\title{
Experimental Removal of Sexual Selection Reveals Adaptations to Polyandry in Both Sexes
}

\section{Journal Article}

\section{Author(s):}

Demont, Marco; Grazer, Vera M.; Michalczyk, Łukasz; Millard, Anna L.; Sbilordo, Sonja H.; Emerson, Brent C.; Gage, Matthew J.G.; Martin, Oliver Y.

\section{Publication date:}

2014-03

\section{Permanent link:}

https://doi.org/10.3929/ethz-b-000080800

\section{Rights / license:}

In Copyright - Non-Commercial Use Permitted

\section{Originally published in:}

Evolutionary biology 41(1), https://doi.org/10.1007/s11692-013-9246-3

\section{Funding acknowledgement:}

137514 - Evolutionary Conflicts and their Impact on Speciation (follow-up) (SNF) 


\title{
Experimental Removal of Sexual Selection Reveals Adaptations to Polyandry in Both Sexes
}

\author{
Marco Demont • Vera M. Grazer • Lukasz Michalczyk • \\ Anna L. Millard · Sonja H. Sbilordo · Brent C. Emerson • \\ Matthew J. G. Gage $\cdot$ Oliver Y. Martin
}

Received: 31 January 2013/Accepted: 1 July 2013/Published online: 12 July 2013

(C) Springer Science+Business Media New York 2013

\begin{abstract}
Polyandrous mating is extremely common, yet for many species the evolutionary significance is not fully resolved. In order to understand the evolution of mating systems, it is crucial that we investigate the adaptive consequences across many facets of reproduction. We performed experimental evolution with the naturally polygamous flour beetle Tribolium castaneum subjected to either polyandry or enforced monogamy, creating contrasting selection regimes associated with the presence or absence of sexual selection. After 36 generations, we investigated male and female adaptations by mating beetles with an unselected tester strain to exclude potential effects of male-female coevolution. Reproductive success of focal monogamous and polyandrous beetles from each sex was assessed in separate single male and multiple male experiments emulating the different selection backgrounds. Males and females from the
\end{abstract}

Marco Demont and Vera M. Grazer have contributed equally to this study.

M. Demont · V. M. Grazer · S. H. Sbilordo · O. Y. Martin ( $\square)$ Experimental Ecology, Institute of Integrative Biology (IBZ),

ETH Zürich, Universitätsstrasse 16, CHN J 11, 8092 Zurich, Switzerland

e-mail: oliver.martin@env.ethz.ch

Ł. Michalczyk

Department of Entomology, Institute of Zoology, Jagiellonian University, Gronostajowa 9, 30-387 Kraków, Poland

A. L. Millard · B. C. Emerson - M. J. G. Gage School of Biological Sciences, University of East Anglia, Norwich NR4 7TJ, UK

B. C. Emerson

Island Ecology and Evolution Research Group (IPNA-CSIC), C/Astrofísico Francisco Sánchez 3, 38206 La Laguna, Tenerife, Canary Islands, Spain polyandrous regime had more offspring in the experiments with multiple males present than monogamous counterparts. However, in single male experiments, neither females nor males differed between selection regimes. Subsequent mating trials with multiple males suggested that adaptations to polyandry in both sexes provide benefits when choice and competition were allowed to take place. Polyandrous females delayed the first copulation when given a choice of males and polyandrous males were quicker to achieve copulation when facing competition. In conclusion, we show that the expected benefits of evolutionary adaptation to polyandry in T. castaneum depended on the availability of multiple mates. This context-dependent effect, which concerned both sexes, highlights the importance of realistic competition and choice experiments.

Keywords Experimental evolution - Mating system . Monogamy · Multiple mating · Reproductive success · Tribolium castaneum

\section{Introduction}

Across the animal kingdom, females commonly mate with more than one male although this is not strictly necessary to cover fertilization needs. Polyandrous mating is especially puzzling given that mating is often associated with considerable costs, such as mechanical damage caused during copulation (Blanckenhorn et al. 2002) or harmful substances transferred with the ejaculate (Chapman et al. 1995; Wigby and Chapman 2005). Although polyandry is widespread in nature, the underlying evolutionary causes often remain elusive and may differ for various species (Jenni 1974; Zeh and Zeh 1996; Zeh and Zeh 1997; Arnqvist and Nilsson 2000; Jennions and Petrie 2000; 
Arnqvist and Rowe 2005; Simmons 2005). Therefore, several evolutionary explanations for polyandry have been proposed to date. According to these, polyandry is variously driven by direct material (e.g. Vahed 1998; Hosken and Stockley 2003), or indirect genetic benefits (Jennions and Petrie 2000; Simmons 2001; Zeh and Zeh 2001; Neff and Pitcher 2005; but see also Kotiaho and Puurtinen 2007), avoiding costs of not remating (Thornhill and Alcock 1983), or via non-adaptive routes (e.g. Halliday and Arnold 1987). Furthermore, it has been suggested that female polyandry might provide a means to combat the negative consequences of inbreeding (Tregenza and Wedell 2002; Firman and Simmons 2008; Michalczyk et al. 2011a) or mating with males bearing selfish genetic elements (Zeh and Zeh 1996; Price et al. 2008).

Nevertheless, there are various obstacles to progress on the way to fully understanding evolutionary causes and consequences of polyandry in many species. At the individual level, there is still a lack of knowledge concerning meaningful natural levels of polyandry and resulting impacts on males and females. In recent studies, this gap has partly been addressed via the use of molecular methods enabling quantification of natural mating rates and sperm utilization (e.g. Bretman and Tregenza 2005; Simmons et al. 2007; Demont et al. 2011, 2012). At a larger scale, we also do not fully understand the adaptations in reproductive traits involved in male-female coevolution associated with having multiple mates. Although polyandry enables sexual selection to occur (via mate choice and competition) it also provides fertile ground for sexual conflicts over all facets of reproduction to germinate. The interests of the sexes are very frequently in opposition (Parker 1979; Arnqvist and Rowe 2005), thus, polyandry can drive adaptations and counter-adaptations to evolve as each sex is selected to gain an edge in this conflict (Chapman et al. 2003; Martin and Hosken 2003a, b; Wigby and Chapman 2004). Therefore, sexual selection and sexual conflict may drive specific adaptations to the polyandrous mating system in females, males or both sexes.

Experimental evolution is a particularly powerful tool for the study of male-female co-evolution and responses to sexual conflict, and has been put to good use in a number of species and contexts (reviewed in Arnqvist and Rowe 2005; Edward et al. 2010). Across previous evolution experiments, contrasting sexual selection/conflict intensities have been implemented in a variety of ways, such as population size/density (e.g. Martin and Hosken 2003b, 2004b; Gay et al. 2009; Hosken et al. 2009), or through contrasting sex ratios (e.g. Wigby and Chapman 2004; Crudgington et al. 2005). Experimental evolution using regimes with different population sex ratios has recently been used in Tribolium castaneum to expose evidence for sexual conflict in this system (Michalczyk et al. 2011b).
Another particularly clear means of achieving contrasting selection regimes is to apply monogamy versus polyandry. Under monogamy with random mate allocation, sexual selection and conflict are essentially absent (Rice 2000), so, one can assess the presence of these selection pressures via an intuitive yes-no dichotomy (e.g. Holland and Rice 1999; Hosken et al. 2001; Hosken and Ward 2001; Martin and Hosken 2003a; Martin et al. 2004).

Tribolium castaneum is naturally highly promiscuous, and direct costs to females of mating multiply are rather low (reviewed in Fedina and Lewis 2008). Hence, this organism is highly suited to investigate potential adaptations to polyandry. Here we assess reproductive success of males and females from monogamous versus polyandrous experimental evolution lines when subjected to single male versus multiple male scenarios to investigate how selection has shaped male and female traits. We focus on two main questions: a) Are polyandrous line beetles generally fitter, perhaps due to good genes effects of sexual selection, or less fit, reflecting costs of sexual conflict? b) Are animals from contrasting selection backgrounds better adapted to equivalent scenarios, i.e. are monogamous beetles fitter when assessed as monogamous pairs, whilst polyandrous beetles are better when multiple males are present? In addition, we assess male and female mating behaviours in the presence of choice and competition, as well as longevity as a measure of general vigour.

\section{Methods}

\section{Experimental Evolution Lines}

Tribolium castaneum is an eminent model system for studies of pre- and postcopulatory sexual selection, characterized by high mating and remating rates (reviewed in Fedina and Lewis 2008). As the source population for the experimental evolution lines described below, we used the $T$. castaneum wild type strain Georgia 1 (Ga1, initial collection 1980, maintained in culture by the Beeman lab at USDA, Manhattan, Kansas). Experimental evolution lines (initiated in 2005 at the University of East Anglia) and other experimental animals were kept on organic white flour supplemented with $10 \%$ brewer's yeast at $30{ }^{\circ} \mathrm{C}$ and ca. $65 \% \mathrm{RH}$. To achieve contrasting sexual selection intensities, we established the following two regimes: monogamy (=sexual selection absent) and polyandry (=sexual selection present). Both treatments consisted of three replicate lines each, i.e. $\mathrm{M} \mathrm{A} / \mathrm{B} / \mathrm{C}$ and $\mathrm{P} \mathrm{A} / \mathrm{B} / \mathrm{C}$. Effective population sizes in each line and in both treatments were estimated as $N_{e}=40$. This is comparable to the $N_{e}=36$ used in related experimental evolution lines using the same source population (see Michalczyk et al. 2011b; Hangartner et al., in press), as well 
as population sizes in similar experiments (see Snook et al. 2009 for an overview). Each monogamy line was founded and then propagated every generation using 20 individually housed beetle pairs. Each polyandry line was founded and propagated using 12 individual females each simultaneously exposed to five different males. Each generation, every pair or group of beetles was housed for seven to ten days (NB same period for all lines per generation) in separate $5 \mathrm{~cm}$ Petri dishes with $10 \mathrm{~g}$ flour-yeast mix and oat flakes on the surface. Food was provided in excess to ensure that density effects were negligible. After this mating period, beetles were discarded, and all larvae were pooled per selection line by collecting the flour in large pots supplemented with additional flour (total volume ca. $500 \mathrm{ml}$ ). In this way, we ensured that the generations were not overlapping. To start each new generation per line, pupae were collected at random from each of the six pools. The pupae were sexed and then kept separated by sex in groups of 15-20 beetles with access to food ad libitum. Pairs and groups for mating were formed with mature beetles at least ten days after collecting pupae. Experimental beetles for the experiments described below were all derived from these ongoing lines in our lab, but from different generations as indicated. Enforcing monogamy without mate choice over a very long time period should potentially remove adaptations to the naturally occurring polygamous mating system, particularly if such adaptations are costly (see e.g. Holland and Rice 1999).

\section{Female RS in Single and Multiple Male Experiments}

To ensure virginity, pupae from the $36^{\text {th }}$ generation were collected and separated by sex. Females from the selection lines were allowed to mate with tester males (i.e. from the unselected source population Ga1), in order to investigate female adaptations in absence of the coevolved male traits, in contrasting single male or multiple male experiments. In the single male experiment, focal females were each maintained with one tester male ( $n=24$ females per line), and in the multiple males experiment females were each housed with five tester males $(n=20$ females per line). These experimental conditions were chosen to mirror the mating conditions during the $\mathrm{M}$ and $\mathrm{P}$ selection history, and allow us to investigate how selected females respond to their own versus opposite conditions. All females were allowed to mate and lay eggs for eight days in $5 \mathrm{~cm}$ diameter Petri dishes containing ca. $10 \mathrm{~g}$ standard flour-yeast mix, topped with organic rolled oats. Then adults were removed and offspring incubated for an additional 29 days before counting reproductive output.

\section{Male RS in Single and Multiple Male Experiments}

Focal males' RS was also assessed with tester beetles in order to investigate male adaptations to polyandry without the interacting effects due to coevolved females. Therefore, using the males from generation 36 , we measured male RS in a single male experiment and paternity share in a multiple males experiment $(n=12-17$ males per line and experimental scenario). Specifically, in the single male experiment, focal males from the selection lines were allowed to reproduce with one tester Ga1-female for 8 days. In the multiple males experiment, a focal male was given access to one Ga1-female, but was in competition with four $R d$-males ( $R d$ : Reindeer, easily identifiable phenotypic marker homozygous in a dominant $R d$ allele, stock supplied by the Beeman lab), so that sex ratios were $\mathrm{F}: \mathrm{M}=1: 5$ as in the experiment from the female's perspective. Here the response measured was the share of paternity accrued by the focal male (=proportion of wild type offspring). Rd has been used successfully with Ga1 in previous experiments (see e.g. Michalczyk et al. 2010, 2011b; Sbilordo et al. 2011). Hence the expected paternity share of focal males in absence of biasing mechanisms would be $20 \%$. After removing the adults after the mating period, offspring were incubated for 29 days before counting reproductive output.

\section{Virgin Longevity Under Starvation}

Twenty-five pupae per sex and selection line were collected from the $37^{\text {th }}$ generation to determine the longevity of virgin beetles under starvation as a measure of general vigour. All pupae were kept isolated in single wells of 96-well plates without access to flour for emergence and also thereafter. Beetles were checked daily to record time until death after emergence. In our analysis, we only used beetles, which had emerged successfully as adults. Longevity was assessed in virgins, as mating activity is known to dramatically decrease female and male longevity in other insect species (e.g. Blanckenhorn et al. 2002; Martin and Hosken 2004a). Furthermore, unmated beetles were preferred, as our selection regimes may be expected to alter such traits as female resistance to male-induced harm (see e.g. Martin and Hosken 2003b, 2004b; Michalczyk et al. 2011b). Starvation was deemed necessary, as this stressful treatment should facilitate the detection of subtle longevity effects (see e.g. Hoffmann and Parsons 1991; Moret and Schmid-Hempel 2000; Martin and Hosken 2003b; Schwarzenbach and Ward 2007). The same assay has successfully been used in a previous experiment using the same beetle stock (Ga1: see Michalczyk et al. 2011a).

\section{Mating Behaviours}

In $T$. castaneum, females discriminate males based on chemical cues and this can bias subsequent copulation and sperm use (Lewis and Austad 1994; Fedina and Lewis 2007). 
It has also been shown that male mating behaviours respond to increased sexual selection intensity applied via experimental evolution in this system (see Michalczyk et al. 2011b). Therefore, female and male mating behaviours could potentially drive differences in RS. Focal beetles for the behaviour experiments were collected from generation 53 as pupae, separated by sex and maintained in same-sex groups (15-20 beetles per $5 \mathrm{~cm}$ Petri dish) until use in experiments. At the same time, test beetles were collected from the Ga1 stock. After emergence, test beetles were marked on the thorax with different colours to be able to discriminate each individual during mating trials. Focal individuals were unmarked. As in the RS experiment, mating trials were conducted separately for males and females. However, behaviours were only measured in an experiment with multiple males to follow up on differences between $\mathrm{M}$ and $\mathrm{P}$ detected in the $\mathrm{RS}$ experiment. In contrast to the experiment on RS, tester males in our mating trials were all Ga1 and not Rd, because here it was not the aim to discriminate offspring from different fathers. Female trials were set up with one focal $\mathrm{M}$ or $\mathrm{P}$ female and three differently marked Gal tester males, whereas male trials consisted of one focal $\mathrm{M}$ or $\mathrm{P}$ male with one tester Ga1 female and two Ga1 competitor males. All beetles were virgins and at least seven days post emergence when the experiments started. On day one of the experiment, groups were put together in a $5 \mathrm{~cm}$ Petri dish with filter paper on the bottom and initially without access to food. The first $30 \mathrm{~min}$ of interactions were observed at room temperature $\left(22-23{ }^{\circ} \mathrm{C}\right)$. We recorded all behaviours where the focal individual was involved, such as the time of the first mount, all subsequent mounts and all copulation durations. Mounting and copulating can be discriminated easily in this species, because the female needs to lift the abdomen for the male to be able to reach the female genital opening with the aedeagus in order to copulate. After the first trials, a small amount of flour-yeast mix was added to each Petri dish. All groups were observed again for $30 \mathrm{~min}$ on the third and sixth day, when beetles were potentially more experienced than on the first day as virgins. Twentyfour beetles per line and for both male and female perspective were observed in total.

\section{Statistical Analyses}

We analysed male and female RS (number of offspring) using nested generalized linear mixed models (i.e. glmer function of lme4 package) in $\mathrm{R}$ version 2.13.0 ( $\mathrm{R}$ Development Core Team 2011). We used the Poisson distribution (log link) for count data. Female RS of the two experiments with one or five males was analysed in one model with selection history ( $\mathrm{M}$ vs. P), environment (one male vs. five males) and their interaction as explanatory variables (fixed factors). Because of the two different response variables for male $\mathrm{RS}$, number of offspring from the single male experiment and paternity share from the multiple males experiment were analysed in separate models and including only selection history as fixed factor. In the model on paternity shares we used the binomial distribution for proportions. Line was included as a random factor nested in selection history in all models. We show $P$ values from the Chi squared distribution obtained with the function Anova() from the car package using type III sum of squares for unequal sample sizes.

The response variables longevity, time to the first mount, time to the first copulation, mean latency per copulation, mean copulation duration, total number of mounts on the female and total number of copulations were analysed using nested linear mixed models (i.e. lmer function of lme4 package). The following transformations were used: longevity squared, log of time to the first mount, time to the first copulation and number of copulations, and square root of latency per copulation, mean copulation duration and total number of mounts. Selection history was the explanatory fixed factor and line was used as a nested random factor. In the analysis of longevity, sex was used as additional fixed factor including its interaction with selection history. $P$ values were obtained as stated before. The residuals of all models fulfilled the model assumptions.

\section{Results}

\section{Female Reproductive Success}

The significant interaction between selection history and environment $\left(\chi_{1}^{2}=6.28, P=0.012\right)$ shows that in the single male experiment (focal female and one tester male) $\mathrm{M}$ - and P-females produced similar numbers of offspring on average (Fig. 1a). In contrast, in the multiple males experiment (focal female and five tester males; Fig. 1b) females with $\mathrm{P}$ selection history had more offspring than females with $\mathrm{M}$ selection history (selection history: $\chi_{1}^{2}=13.99, \quad P<0.001$; environment: $\left.\chi_{1}^{2}=1.68, P=0.195\right)$.

\section{Male Reproductive Success}

In the single male experiment (one tester female and focal male), the total number of offspring produced was not influenced by the male's selection history $\left(\chi_{1}^{2}=0.21\right.$, $P=0.649$; Fig. 2a). In contrast, in the multiple males experiment (one tester female and focal male and four competitor marker males), the paternity share of the focal male was significantly influenced by selection history $\left(\chi_{1}^{2}=27.32, P<0.001\right.$; Fig. 2 b). If all five competing males in each assay were equal, and pre- and 

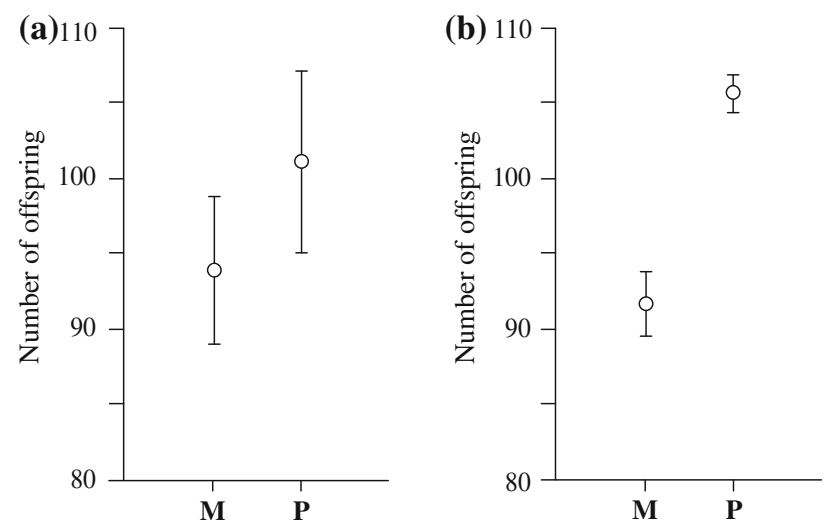

Fig. 1 Female reproductive success of three monogamous $(M)$ versus three polyandrous $(P)$ selection lines (means $\pm 1 \mathrm{SE}$ ). Females ( $\mathrm{n}=19-24$ per line) were exposed to single or multiple tester males to investigate adaptations to the presence of sexual selection in absence of effects due to coevolved males. a Single male experiment: focal female and one tester male for eight days. b Multiple males experiment: focal female and five tester males for eight days
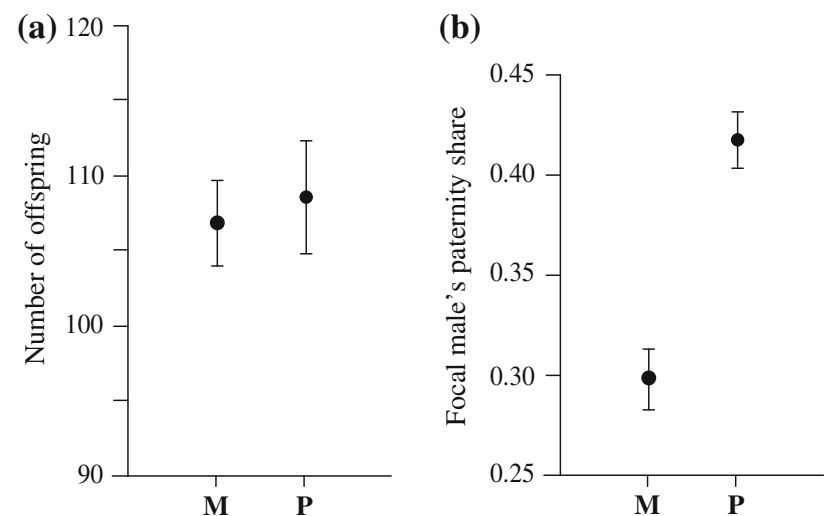

Fig. 2 Male reproductive success and paternity shares from three monogamous $(M)$ versus three polyandrous $(P)$ selection lines (means $\pm 1 \mathrm{SE})$. Males $(\mathrm{n}=12-17$ per line) were assessed in a single male experiment (i.e. no competition over access to female) or a multiple males experiment (i.e. competitor males present) to investigate adaptations to the presence of sexual selection in absence of effects due to coevolved females. a Single male experiment: focal male and one tester female for eight days. b Multiple males experiment: focal male and one tester female and four competitor Rd males for eight days

postcopulatory competition mechanisms were absent, one would expect a $20 \%$ paternity share for the focal male, regardless of selection history. However, M-males were only slightly above this value with an average share of $30 \%$, whereas on average P-males sired $42 \%$ of all offspring in competition with four marker males.

\section{Virgin Longevity Under Starvation}

M-females lived on average $24 \pm 2$ days (i.e. line mean $\pm 1 \mathrm{SE} ; \mathrm{n}=20-24$ per line) and M-males $23 \pm 2$ days

( $\mathrm{n}=22-24$ per line). Males $(\mathrm{n}=23-25$ per line $)$ and females $(\mathrm{n}=24$ per line) from P-lines lived on average $26 \pm 1$ days and $27 \pm 1$ days (means $\pm 1 \mathrm{SE}$ ). Based on AIC, the non-significant interaction was removed from the model. There were no differences in longevity between the sexes $\left(\chi_{1}^{2}=2.43, \quad P=0.119\right)$ or selection histories $\left(\chi_{1}^{2}=2.39, P=0.123\right)$.

\section{Mating Behaviours from the Female Perspective}

There was no difference in the time elapsed until a M- or P-female was mounted for the first time $\left(\chi_{1}^{2}=3.07\right.$, $P=0.080$; Fig. 3), except a weak trend that P-females were mounted faster. P-females took significantly longer to copulate for the first time than $\mathrm{M}$-females $\left(\chi_{1}^{2}=5.60, P=0.018\right.$; Fig. 3). Over all three observation time points, however, females with $\mathrm{M}$ or $\mathrm{P}$ selection history were not different in terms of copulation latency after mounting (line mean \pm SE: M: $\left.11.1 \pm 0.2 \mathrm{~s}, \mathrm{P}: 14.7 \pm 4.0 \mathrm{~s}, \chi_{1}^{2}=0.13, P=0.721\right)$, mean copulation duration $(\mathrm{M}: 75 \pm 8 \mathrm{~s}, \mathrm{P}: 67 \pm 5 \mathrm{~s}$, $\left.\chi_{1}^{2}=0.93, P=0.335\right)$ or the total number of copulations (M: $1.8 \pm 0.1, \mathrm{P}: 1.4 \pm 0.2, \chi_{1}^{2}=0.91, P=0.340$ ).

Mating Behaviours from the Male Perspective

On average M- and P-males waited equally long until they mounted the tester female for the first time $\left(\chi_{1}^{2}=1.15\right.$, $P=0.283$; Fig. 4 ), but with P-males the first copulation started earlier than with M-males $\left(\chi_{1}^{2}=5.63, P=0.018\right)$. Considering all three observation periods, there were no significant differences between selection histories in the

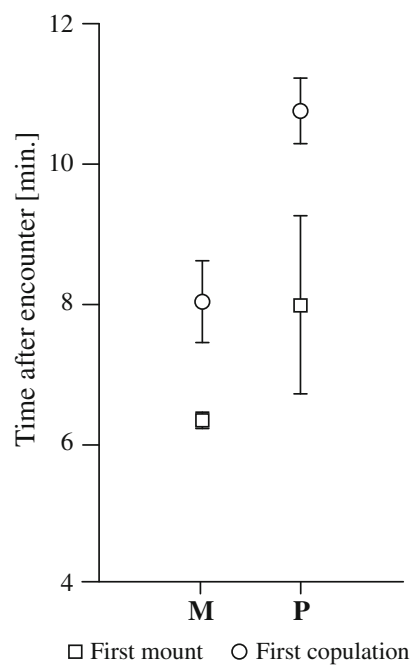

Fig. 3 Female mating behaviours. Focal females from monogamous $(M)$ and polyandrous $(P)$ selection regimes observed in mating trials with three tester males. All beetles were virgins at the first encounter. Here, first mount $=$ female being mounted for the first time. Data points represent means \pm 1 SE over three lines $(n=15-23$ per line $)$ 


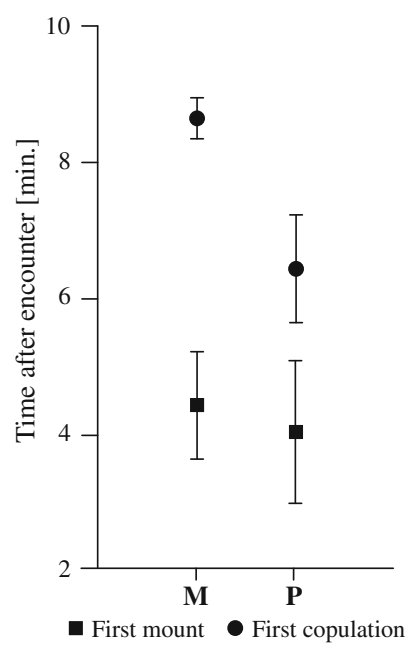

Fig. 4 Male mating behaviours. Focal males from monogamous $(M)$ and polyandrous $(P)$ selection regimes observed in mating trials with one tester female and two competitor tester males. All beetles were virgins at the first encounter. Data points represent means \pm 1 SE of three lines $(n=13-24$ per line $)$

total number of mounts on the female (line mean \pm SE: M: $4.3 \pm 0.7, \quad$ P: $\left.4.8 \pm 1.1, \quad \chi_{1}^{2}=0.02, \quad P=0.888\right)$, total number of copulations $(\mathrm{M}: 1.7 \pm 0.4, \quad \mathrm{P}: 1.9 \pm 0.5$, $\left.\chi_{1}^{2}=0.04, P=0.832\right)$ and mean copulation duration $(\mathrm{M}$ : $\left.1.7 \pm 0.2 \mathrm{~min}, \mathrm{P}: 1.8 \pm 0.1 \mathrm{~min}, \chi_{1}^{2}=0.00, P=0.961\right)$.

\section{Discussion}

Using contrasting mating experiments, we revealed context-dependent adaptations to polyandry in both sexes of our model organism T. castaneum. When assessed in single pairs, so in the absence of mate choice and competition, monogamous and polyandrous beetles achieved equal reproductive fitness. However, when multiple males were present, males and females from the polyandrous regime both had more offspring than their monogamous counterparts. Because males and females were assayed in separate experiments, these results show that polyandrous individuals of both sexes can gain reproductive benefits under their naturally promiscuous mating regime. This mirrors previous work on Scathophaga stercoraria indicating that polyandrous animals require multiple mates in order to profit from their adaptations (Martin et al. 2004). Behavioural assays indicate that polyandrous females delay their first copulation compared to monogamous females when multiple males are present. Additionally, polyandrous males were more efficient in obtaining copulations swiftly following mounting. Adding to the detection of sexual conflict in this species by Michalczyk et al. (2011b), by enforcing strict monogamy, here, experimental evolution revealed independent adaptations to polyandry in $T$. castaneum males and females.

Female Adaptation to Polyandry

Females with polyandrous background may have higher reproductive success in the presence of multiple males if particular adaptations provide benefits of sexual selection due to polyandry (reviewed e.g. in Jennions and Petrie 2000; Zeh and Zeh 2001). Specifically, they could have higher mating and remating rates thereby increasing not only sperm supply (direct benefit) but also the chance and strength of sperm competition (indirect benefits; Arnqvist and Nilsson 2000). Likewise, polyandrous females may be better than monogamous females at making the right mating choice(s) to pick the optimally suited father(s) to gain indirect genetic benefits for their offspring (Tregenza and Wedell 2000; Mays and Hill 2004). It has been shown that in stock $T$. castaneum females, access to multiple males is neither beneficial nor costly under standard conditions, with no detectable effect on female fitness (Pai and Yan 2003; Grazer and Martin 2012). In contrast, after a rapid temperature increase of $4{ }^{\circ} \mathrm{C}$ relative to the standard temperature, females in the experiment with multiple males achieved higher reproductive success than females in the experiment with a single male, suggesting that environmental change can shift the balance between costs and benefits of multiple mating (Grazer and Martin 2012). In this previous experiment, which used the source population of the present experimental evolution study, the most likely explanation for the observed pattern seemed to be sexual selection for good or compatible genes in the novel environment (reviewed in Mays and Hill 2004; Grazer and Martin 2012). In order to investigate which exact mechanisms are responsible for polyandrous females profiting more from multiple males it is necessary to have information concerning mating propensities and mating rates. Our behavioural assays indicate that when faced with multiple males, polyandrous females take longer to copulate for the first time, but do not differ from monogamous females in terms of number of copulations or copulation duration. This might suggest that the difference in offspring does not stem from polyandrous females having access to a greater quantity of sperm than monogamous females. On the contrary, polyandrous females exposed to a multiple male scenario appear to be choosier. In $T$. castaneum, the female is able to delay or even resist copulations by lowering the abdomen or moving around rapidly, such that the mounted male cannot reach the genital opening with the aedeagus. Furthermore, competitor males have frequently been observed to interact with mounted pairs to bring down the mounted male and to take over the female (personal observations, VMG and OYM). Therefore, considering 
species biology, polyandrous females might obtain reproductive benefits from waiting for the suitable male rather than mating with multiple males when given a choice.

In contrast, our findings for female fitness may not be driven by benefits of sexual selection. It has frequently been shown that polyandrous females may evolve to deal better with mating costs due to sexual conflict (Arnqvist and Rowe 2005). In particular, experimental evolution studies have been very useful for revealing hidden costs (e.g. Rice 1996; Holland and Rice 1999; Martin and Hosken 2003a; Martin et al. 2004). A previous experimental evolution study on $T$. castaneum applying different population sex ratios rather than contrasting mating systems demonstrated that experimental manipulation may be necessary to capture clear evidence of sexual conflict (Michalczyk et al. 2011b). Females exposed to weak sexual selection intensity (i.e. female-biased sex ratio, contrasted vs. male-biased, high conflict regime) suffered more from exposure to multiple males (Michalczyk et al. 2011b). When exposed to increasing numbers of (control) males, females from female-biased lines showed a steep decline in reproductive fitness (Michalczyk et al. 2011b). This finding seemed to be a response to increased levels of mating vigour and thus harmfulness of males from male-biased selection lines (Michalczyk et al. 2011b). A recent study identified a range of seminal fluid proteins in T. castaneum, which are transferred to the female reproductive tract (South et al. 2011). Although it is yet unknown whether any of these proteins have costs for females there might be at least the possibility of finding similar negative effects of postcopulatory sexual conflict as in Drosophila melanogaster (Chapman et al. 1995; Wigby and Chapman 2005). The female behaviours assessed here, however, did not indicate that polyandrous females are under selection to reduce mating frequency, mating duration or increase resistance to male mating attempts. Nevertheless, further work would be needed to elucidate which precopulatory and postcopulatory mechanisms drive the improved performance of polyandrous line females under polyandry.

In sum, improved reproductive success of females under polyandry does not seem to be due to mating more frequently or longer. Rather differences seem to relate to a higher latency to the first copulation, potentially due to increased choosiness of the female or in order to reduce mating costs.

\section{Male Adaptation to Polyandry}

Similar to our findings for females, we find that in the single male experiment (focal male housed with one tester female) our measure of reproductive success was not influenced by selection history: monogamous and polyandrous males produced equal numbers of offspring. This finding contrasts with other studies where polyandrous individuals were found to be fitter regardless of the number of available mates (e.g. Pitnick et al. 2001; Crudgington et al. 2005). In the multiple males experiment, where focal males were forced to compete with four marker males (i.e. Reindeer mutant) for access to a single tester female (i.e. from Ga1 stock), the focal male's paternity share was significantly influenced by male selection history. Polyandrous males had a significantly greater share of paternity $(42 \%)$ than expected without sexual selection mechanisms (i.e. $20 \%$ ) compared to their monogamous counterparts $(30 \%)$. This finding of superior male competitive ability mirrors findings in previous experiments (e.g. Hosken et al. 2001; Pitnick et al. 2001; Simmons and Garcia-Gonzalez 2008). These results might be driven by differences in general vigour across regimes; however, we found no differences in male longevity under starvation. Rather, our observations of behaviour indicate that the quite substantial difference in paternity shares is at least to some extent due to precopulatory processes. We found that, in the face of competition with unselected tester males (i.e. from $\mathrm{Ga} 1$ stock), polyandrous males were quicker to copulate than monogamous males, although they were not quicker to mount. This hence indicates greater efficiency at obtaining matings. Further support for this notion comes from a related experimental evolution study applying different sex ratios to the same source population. There, males from male-biased sex ratios (i.e. exposed to increased sexual selection intensity) obtained greater reproductive success when forced to compete for females with a tester male than males from a female-biased background (Michalczyk et al. 2011b). Furthermore, this difference seemed to relate to precopulatory male behaviour as well, as these males were faster to mount females and spent more time mounting and mating (Michalczyk et al. 2011b). Whereas varying sexual selection intensity via population sex ratios as in Michalczyk et al. (2011b) allows the study of a range of sexual selection intensities, the use of a monogamy treatment as done here effectively removes choice and competition from the environment beetles evolved under. In the present study, the contrast is hence even clearer, as we can compare situations with versus without sexual selection in a dichotomous manner (see also Grazer and Martin 2012). Overall, it is likely that males from the polyandrous regime (i.e. where precopulatory and postcopulatory sexual selection and conflict mechanisms were able to act) were not only better in precopulatory mate competition, but also in sperm competition. Further experiments should investigate whether differences in reproductive success are also due to, for example, higher sperm numbers or improved sperm competitiveness of polyandrous males. Contrasting with the more subtle results in females (potentially increased choosiness), superior fitness of polyandrous males in multiple male situations appears to be a more direct reflection of improved male 
competitive ability. Interestingly, though, a multiple male scenario is necessary to expose this.

\section{Conclusions}

In separate experiments, we found that both males and females from polyandrous lines had greater reproductive success when multiple males are present (i.e. competition and choice present) than monogamous counterparts. There was no indication that adaptations responsible for this difference bear large costs in either sex, at least within the confines of our experiments. Reproductive output of monogamous and polyandrous line animals was equal when assessed monogamously (i.e. mate choice and competition absent). Furthermore, polyandrous animals were not simply better in both single male and multiple male scenarios, so are not generally fitter. Monogamous animals were not superior under monogamy, so there is also no indication of specific adaptation to monogamy. Assays of mating behaviour in both sexes when multiple males were present indicate that polyandrous females delayed their first copulation while polyandrous males achieved copulations more quickly. Our findings underline how differential sexual selection intensity moulds the evolution of reproductive traits and fitness. Finally, and strikingly, adaptations to polyandry in both sexes only became apparent when selecting mechanisms were allowed to act in the presence of multiple males.

Acknowledgments We thank the Swiss National Science Foundation (Ambizione Grants PZ00P3_121777 and PZ00P3-137514 and standard research Grant 31003A_125144/1 to OYM), ETH Zurich, NERC and the University of East Anglia for support. We further thank Helen Alexander and the anonymous reviewers for helpful comments. Finally, we are very grateful to Richard Beeman (USDA) for providing the beetle stock originally used to generate the selection lines assessed here.

\section{References}

Arnqvist, G., \& Nilsson, T. (2000). The evolution of polyandry: Multiple mating and female fitness in insects. Animal Behaviour, 60, 145-164.

Arnqvist, G., \& Rowe, L. (2005). Sexual conflict. Princeton: Princeton University Press.

Blanckenhorn, W. U., Hosken, D. J., Martin, O. Y., Reim, C., Teuschl, Y., \& Ward, P. I. (2002). The costs of copulating in the dung fly Sepsis cynipsea. Behavioral Ecology, 13, 353-358.

Bretman, A., \& Tregenza, T. (2005). Measuring polyandry in wild populations: A case study using promiscuous crickets. Molecular Ecology, 14, 2169-2179.

Chapman, T., Arnqvist, G., Bangham, J., \& Rowe, L. (2003). Sexual conflict. Trends in Ecology \& Evolution, 18, 41-47.

Chapman, T., Liddle, L. F., Kalb, J. M., Wolfner, M. F., \& Partridge, L. (1995). Cost of mating in Drosophila melanogaster females is mediated by male accessory gland products. Nature, 373, 241-244.
Crudgington, H. S., Beckerman, A. P., Brustle, L., Green, K., \& Snook, R. R. (2005). Experimental removal and elevation of sexual selection: Does sexual selection generate manipulative males and resistant females? American Naturalist, 165, S72-S87.

Demont, M., Buser, C. C., Martin, O. Y., \& Bussière, L. F. (2011). Natural levels of polyandry: Differential sperm storage and temporal changes in sperm competition intensity in wild yellow dung flies. Functional Ecology, 25, 1079-1090.

Demont, M., Martin, O. Y., \& Bussière, L. F. (2012). Wild yellow dung fly females may not select sperm based on dung pat microclimate but could nevertheless benefit from polyandry. Evolutionary Ecology, 26, 715-731.

Edward, D. A., Fricke, C., \& Chapman, T. (2010). Adaptations to sexual selection and sexual conflict: Insights from experimental evolution and artificial selection. Philosophical Transactions of the Royal Society B-Biological Sciences, 365, 2541-2548.

Fedina, T. Y., \& Lewis, S. M. (2007). Female mate choice across mating stages and between sequential mates in flour beetles. Journal of Evolutionary Biology, 20, 2138-2143.

Fedina, T. Y., \& Lewis, S. M. (2008). An integrative view of sexual selection in Tribolium flour beetles. Biological Reviews, 83, 151-171.

Firman, R. C., \& Simmons, L. W. (2008). Polyandry facilitates postcopulatory inbreeding avoidance in house mice. Evolution, 62, 603-611.

Gay, L., Eady, P. E., Vasudev, R., Hosken, D. J., \& Tregenza, T. (2009). Does reproductive isolation evolve faster in larger populations via sexually antagonistic coevolution? Biology Letters, 5, 693-696.

Grazer, V. M., \& Martin, O. Y. (2012). Elevated temperature changes female costs and benefits of reproduction. Evolutionary Ecology, 26, 625-637.

Halliday, T., \& Arnold, S. J. (1987). Multiple mating by females-A perspective from quantitative genetics. Animal Behaviour, 35, 939-941.

Hangartner, S. B., Sbilordo, S. H., Michalczyk, Ł., Gage, M. J. G., \& Martin, O. Y. (in press). Are there genetic trade-offs between immune and reproductive investments in Tribolium castaneum? Infection, Genetics and Evolution.

Hoffmann, A. A., \& Parsons, P. A. (1991). Evolutionary genetics and environmental stress. Oxford: Oxford University Press.

Holland, B., \& Rice, W. R. (1999). Experimental removal of sexual selection reverses intersexual antagonistic coevolution and removes a reproductive load. Proceedings of the National Academy of Sciences of the United States of America, 96, 5083-5088.

Hosken, D. J., Garner, T. W. J., \& Ward, P. I. (2001). Sexual conflict selects for male and female reproductive characters. Current Biology, 11, 489-493.

Hosken, D. J., Martin, O. Y., Wigby, S., Chapman, T., \& Hodgson, D. J. (2009). Sexual conflict and reproductive isolation in flies. Biology Letters, 5, 697-699.

Hosken, D. J., \& Stockley, P. (2003). Benefits of polyandry: A life history perspective. Evolutionary Biology, 33, 173-194.

Hosken, D. J., \& Ward, P. I. (2001). Experimental evidence for testis size evolution via sperm competition. Ecology Letters, 4, 10-13.

Jenni, D. A. (1974). Evolution of polyandry in birds. American Zoologist, 14, 129-144.

Jennions, M. D., \& Petrie, M. (2000). Why do females mate multiply? A review of the genetic benefits. Biological Reviews, 75, 21-64.

Kotiaho, J. S., \& Puurtinen, M. (2007). Mate choice for indirect genetic benefits: scrutiny of the current paradigm. Functional Ecology, 21, 638-644.

Lewis, S. M., \& Austad, S. N. (1994). Sexual selection in flour beetles-The relationship between sperm precedence and male olfactory attractiveness. Behavioral Ecology, 5, 219-224. 
Martin, O. Y., \& Hosken, D. J. (2003a). Costs and benefits of evolving under experimentally enforced polyandry or monogamy. Evolution, 57, 2765-2772.

Martin, O. Y., \& Hosken, D. J. (2003b). The evolution of reproductive isolation through sexual conflict. Nature, 423, 979-982.

Martin, O. Y., \& Hosken, D. J. (2004a). Copulation reduces male but not female longevity in Saltella sphondylli (Diptera: Sepsidae). Journal of Evolutionary Biology, 17, 357-362.

Martin, O. Y., \& Hosken, D. J. (2004b). Reproductive consequences of population divergence through sexual conflict. Current Biology, 14, 906-910.

Martin, O. Y., Hosken, D. J., \& Ward, P. I. (2004). Postcopulatory sexual selection and female fitness in Scathophaga stercoraria. Proceedings of the Royal Society of London Series B-Biological Sciences, 271, 353-359.

Mays, H. L., \& Hill, G. E. (2004). Choosing mates: Good genes versus genes that are a good fit. Trends in Ecology \& Evolution, 19, 554-559.

Michalczyk, Ł., Martin, O. Y., Millard, A. L., Emerson, B. C., \& Gage, M. J. G. (2010). Inbreeding depresses sperm competitiveness, but not fertilization or mating success in male Tribolium castaneum. Proceedings of the Royal Society B-Biological Sciences, 277, 3483-3491.

Michalczyk, Ł., Millard, A. L., Martin, O. Y., Lumley, A. J., Emerson, B. C., Chapman, T., et al. (2011a). Inbreeding promotes female promiscuity. Science, 333, 1739-1742.

Michalczyk, Ł., Millard, A. L., Martin, O. Y., Lumley, A. J., Emerson, B. C., \& Gage, M. J. G. (2011b). Experimental evolution exposes female and male responses to sexual selection and conflict in Tribolium castaneum. Evolution, 65, 713-724.

Moret, Y., \& Schmid-Hempel, P. (2000). Survival for immunity: The price of immune system activation for bumblebee workers. Science, 290, 1166-1168.

Neff, B. D., \& Pitcher, T. E. (2005). Genetic quality and sexual selection: an integrated framework for good genes and compatible genes. Molecular Ecology, 14, 19-38.

Pai, A., \& Yan, G. Y. (2003). Rapid female multiple mating in red flour beetles (Tribolium castaneum). Canadian Journal of Zoology-Revue Canadienne De Zoologie, 81, 888-896.

Parker, G. A. (1979). Sexual selection and sexual conflict. In M. S. Blum \& N. A. Blum (Eds.), Sexual selection and reproductive competition in insects (pp. 123-166). London: Academic Press.

Pitnick, S., Miller, G. T., Reagan, J., \& Holland, B. (2001). Males' evolutionary responses to experimental removal of sexual selection. Proceedings of the Royal Society of London Series B-Biological Sciences, 268, 1071-1080.

Price, T. A. R., Hodgson, D. J., Lewis, Z., Hurst, G. D. D., \& Wedell, N. (2008). Selfish genetic elements promote polyandry in a fly. Science, 322, 1241-1243.

Rice, W. R. (1996). Sexually antagonistic male adaptation triggered by experimental arrest of female evolution. Nature, 381, 232-234.

Rice, W. R. (2000). Dangerous liaisons. Proceedings of the National Academy of Sciences of the United States of America, 97, 12953-12955.
Sbilordo, S. H., Grazer, V. M., Demont, M., \& Martin, O. Y. (2011). Impacts of starvation on male reproductive success in Tribolium castaneum. Evolutionary Ecology Research, 13, 347-359.

Schwarzenbach, G. A., \& Ward, P. I. (2007). Phenoloxidase activity and pathogen resistance in yellow dung flies Scathophaga stercoraria. Journal of Evolutionary Biology, 20, 2192-2199.

Simmons, L. W. (2001). The evolution of polyandry: An examination of the genetic incompatibility and good-sperm hypotheses. Journal of Evolutionary Biology, 14, 585-594.

Simmons, L. W. (2005). The evolution of polyandry: Sperm competition, sperm selection, and offspring viability. Annual Review of Ecology Evolution and Systematics, 36, 125-146.

Simmons, L. W., Beveridge, M., \& Kennington, W. J. (2007). Polyandry in the wild: Temporal changes in female mating frequency and sperm competition intensity in natural populations of the Tettigoniid Requena verticalis. Molecular Ecology, 16, 4613-4623.

Simmons, L. W., \& Garcia-Gonzalez, F. (2008). Evolutionary reduction in testes size and competitive fertilization success in response to the experimental removal of sexual selection in dung beetles. Evolution, 62, 2580-2591.

Snook, R. R., Brüstle, L., \& Slate, J. (2009). A test and review of the role of effective population size on experimental sexual selection patterns. Evolution, 63, 1923-1933.

South, A., Sirot, L. K., \& Lewis, S. M. (2011). Identification of predicted seminal fluid proteins in Tribolium castaneum. Insect Molecular Biology, 20, 447-456.

Thornhill, R., \& Alcock, J. (1983). The evolution of insect mating systems. Cambridge: Harvard University Press.

Tregenza, T., \& Wedell, N. (2000). Genetic compatibility, mate choice and patterns of parentage: Invited review. Molecular Ecology, 9, 1013-1027.

Tregenza, T., \& Wedell, N. (2002). Polyandrous females avoid costs of inbreeding. Nature, 415, 71-73.

Vahed, K. (1998). Sperm precedence and the potential of the nuptial gift to function as paternal investment in the Tettigoniid Steropleurus stali Bolivar (Orthoptera: Tettigoniidae: Epphippigerinae). Journal of Orthoptera Research, 7, 223-226.

Wigby, S., \& Chapman, T. (2004). Female resistance to male harm evolves in response to manipulation of sexual conflict. Evolution, 58, 1028-1037.

Wigby, S., \& Chapman, T. (2005). Sex peptide causes mating costs in female Drosophila melanogaster. Current Biology, 15, 316-321.

Zeh, J. A., \& Zeh, D. W. (1996). The evolution of polyandry I: Intragenomic conflict and genetic incompatibility. Proceedings of the Royal Society B-Biological Sciences, 263, 1711-1717.

Zeh, J. A., \& Zeh, D. W. (1997). The evolution of polyandry II: Postcopulatory defences against genetic incompatibility. Proceedings of the Royal Society B-Biological Sciences, 264, 69-75.

Zeh, J. A., \& Zeh, D. W. (2001). Reproductive mode and the genetic benefits of polyandry. Animal Behaviour, 61, 1051-1063. 